\title{
Correlation between Multidrug-Resistant Bacteria Colonization and Bloodstream Infections in Children with Hematolymphoid Malignancies at a Tertiary Cancer Center in India
}

\author{
Nihad Salifu ${ }^{1}$ Gaurav Narula² Maya Prasad $^{2}$ Sanjay Biswas ${ }^{3}$ Rohini Kelkar ${ }^{3}$ Shripad Banavali²
}

${ }^{1}$ Department of Child Health, Korle Bu Teaching Hospital, Accra, Ghana

2Department of Pediatric Oncology, Tata Memorial Hospital, HBNI, Mumbai, Maharashtra, India

${ }^{3}$ Department of Microbiology, Tata Memorial Hospital, Mumbai, Maharashtra, India

Ind J Med Paediatr Oncol 2021;42:61-66.

\begin{abstract}
Address for correspondence Gaurav Narula, MD, DNB, Department of Pediatric Oncology, Tata Memorial Hospital, HBNI, Mumbai, Maharashtra, India (e-mail: drgauravnarula@gmail.com).
\end{abstract}

\begin{abstract}
Keywords

- Bloodstream

- colonization

- enteric

- infections

Background Bloodstream infections with drug-resistant bacteria are associated with a higher morbidity and mortality. Based on previous studies in our institution demonstrating a rising incidence of multidrug resistant organism (MDR) bacteria in bloodstream infections (BSI) and high prevalence of enteric colonization with MDR, the "de-escalation" strategy for empirical antibiotics was adopted in the management of febrile neutropenia in children with hematolymphoid malignancies and MDR colonization. An audit was carried out to determine whether surveillance rectal swabs correlated with blood cultures in febrile neutropenia.

Methods Patient data from January 2015 to July 2016 was examined. Rectal swabs of children with hematolymphoid malignancies were obtained at presentation. Blood cultures were taken during febrile neutropenia. Data were analyzed using SPSS version 24.0. The significance level was set at $p<0.05$.

Results Most patients (62.1\%) with confirmed BSI were colonized with either extended-spectrum $\beta$-lactamase producing organisms (ESBLO) (31.9\%) or MDR (30.2\%). Majority 116 (62.7\%) developed BSI caused by either MDR or ESBLO. In contrast, only $12(10.6 \%)$ patients colonized by sensitive bacteria, developed BSI caused by either MDR or ESBLO. These differences were statistically significant $(p<0.001)$. Thus, the baseline rectal swab had a sensitivity and a specificity of $90.6 \%$ and $59.4 \%$, respectively, in predicting BSI with either MDR or ESBLO.

Conclusions We conclude that high prevalence of MDR colonization at presentation significantly results in MDR BSI, which further results in a significant increase in intensive care unit admissions and mortality. This would justify the use of a "de-escalation" antibiotic policy. Whether such a strategy has been successful in impacting outcomes, would need further study.
\end{abstract}

DOI https://doi.org/ $10.1055 / \mathrm{s}-0041-1730852$ ISSN 0971-5851
(C) 2021. Indian Society of Medical and Paediatric Oncology This is an open access article published by Thieme under the terms of the Creative Commons Attribution-NonDerivative-NonCommercial-License, permitting copying and reproduction so long as the original work is given appropriate credit. Contents may not be used for commercial purposes, or adapted, remixed, transformed or built upon. (https://creativecommons.org/licenses/by-nc-nd/4.0/). Thieme Medical and Scientific Publishers Pvt. Ltd. A-12, 2nd Floor, Sector 2, Noida-201301 UP, India 


\section{Introduction}

Bloodstream infections (BSI) with multidrug-resistant (MDR) bacteria are associated with a higher morbidity and mortality. ${ }^{1}$ Our institution has previously reported a rising incidence in BSI caused by MDR bacteria, a high prevalence of rectal colonization by MDR and extended-spectrum $\beta$-lactamase-producing organisms (ESBLO), and a high conversion rate from sensitive bacteria to resistant strains over time in pediatric patients with cancer. This informed the decision of the pediatric hematolymphoid disease management group to adopt the "de-escalation" strategy of the Expert Group of the 4th European Conference of Infections in Leukemia (ECIL-4) guidelines for empirical antibiotics in the management of patients with febrile neutropenia. ${ }^{2}$

The objectives of this audit were to determine the correlation between enteric colonization with MDR bacteria and BSI and to evaluate the association between colonization with MDR bacteria and intensive care unit (ICU) admission and mortality from infections.

The audit was approved by the Institutional Ethics Committee of the Tata Memorial Hospital with approval number P. No 3325/2019.

\section{Design/Methods}

This audit was conducted in the pediatric oncology unit of a large tertiary cancer care center in western India, delivering services to patients from all over India and other countries in Asia and Africa.

\section{Antibiotic Policy}

The unit utilized the "de-escalation" policy of the ECIL-4 guidelines and prospectively applied it to patients with hematolymphoid malignancies who developed febrile neutropenia. The policy is to start with a combination of colistin and carbapenem on the first sign of fever in neutropenic patients who were known to be colonized with MDR. The higher end of the colistin maximum dose recommended for children was used at 150,000 IU $/ \mathrm{kg}$, divided every 6 hours, and modified when indicated by creatinine clearance. If the patients subsequently improved, and blood culture did not grow any organism or showed an organism with a different sensitivity, the antibiotics would be rapidly "de-escalated" to a more standard first-line combination of use in the unit.

\section{Other Measures to Reduce Colonization}

Other strategies employed by our unit to limit colonization included segregation and cohorting of MDR colonized patients during admissions and use of best infection control policies, including handwash, gowning, and masks when dealing with these patients.

Data prospectively collected from January 2015 to July 2016 was examined. Rectal swabs were obtained by introducing a sterile swab stick into the rectum of patients registered with hematolymphoid malignancies (profile in - Table 1) on day of presentation, or within 7 days if missed on day 1 . Repeat rectal swabs within 15 days were taken to track in cases of "no isolate" on the initial swab. This occurred sometimes as patients had received antibiotics before referral to our center. Others were repeated during febrile neutropenia episodes and admission to evaluate change in flora in previously non-MDR colonized patients who did not show improvement on empirical antibiotics or to evaluate the persistence of MDR strains (this was done only for MDR-positive patients).

Blood cultures were taken during febrile episodes from peripheral veins and central lines when present. Records of all cultures were examined. Sensitivity patterns were studied using the Kirby-Bauer's disc diffusion method. Clinical and Laboratory Standards Institute (CLSI) guidelines were used to select the antibiotic disc. A limited panel was used to determine ESBLO, carbapenem-resistant Enterobacteriaceae (CRE), and vancomycin-resistant enterococci (VRE).

ESBL production was confirmed by CLSI recommendations using cephalosporin-clavulanate combination disks. A difference of $\geq 5 \mathrm{~mm}$ between zone diameter of either of the cephalosporin discs and their respective cephalosporin-clavulanate disc was taken to be phenotypic confirmation of ESBL production. We used cefotaxime (30 $\mu \mathrm{g})$, ceftazidime (30 $\mu \mathrm{g})$, and ceftazidime/clavulanic acid ( $30 \mu \mathrm{g} / 10 \mu \mathrm{g})$ disks for ESBL determination. Carbapenem resistance was reported as per the CLSI guidelines. Vancomycin resistance was confirmed by minimal inhibitory concentrations with the "E" test. In an event when more than one organism with differing sensitivity profile was isolated from the culture, the worse sensitivity pattern was taken into account for analysis.

\section{Definition of Resistance}

MDR was defined as nonsusceptibility to at least a carbapenem, polymyxin (colistin), and an antipseudomonal cephalosporin (ceftazidime). ${ }^{3}$ Nonsusceptibility to a third-generation cephalosporin or in combination with a clavulanate but not any of the agents mentioned in defining MDR delineated ESBLO. ${ }^{4}$ ESBLO intermediate strains were considered to be resistant.

\section{Statistical Methods Used}

We analyzed the data of children with hematolymphoid malignancies who developed BSI from January 2015 to July 2016. We correlated their blood culture results during the episodes of febrile neutropenia with their baseline rectal colonization before starting therapy. Finally, we determined the outcome of the febrile neutropenia episodes in terms of ICU transfer and death. Simple descriptive statistics were used to present the demographic, clinical characteristics, details of baseline rectal colonization, and BSI of the patients. Paired data were analyzed to test strength of the association of MDR colonization with MDR BSI and outcome parameters such as ICU admissions and mortality using a two-sided Pearson Chi-square. Statistical analysis was conducted using SPSS version 24.0, and significance level was set at $p<0.05$ with a confidence interval of $95 \%$. 
Table 1 Profile of patients, baseline rectal swabs, and BSI (1094 patients)

\begin{tabular}{|c|c|}
\hline Variable & $n(\%)$ \\
\hline Male & $743(68)$ \\
\hline Female & $351(32)$ \\
\hline Male:female ratio & $2.1: 1$ \\
\hline Median age (range) & 7 years (6 months -15 years) \\
\hline ALL & $688(62.9)$ \\
\hline AML & $157(14.4)$ \\
\hline $\mathrm{NHL}$ & $124(11.4)$ \\
\hline $\mathrm{HL}$ & $102(9.3)$ \\
\hline MDS & $10(0.9)$ \\
\hline CML blast/accelerated phase & $13(1.2)$ \\
\hline Total number of patients colonized with two different bacteria at presentation & 191 \\
\hline Total number of patients colonized with three different bacteria at presentation & 35 \\
\hline Total number of organisms isolated at presentation & 997 \\
\hline Number of patients with $E$. coli & 680 \\
\hline enteric colonization & ( $62 \%$ of total number of patients) \\
\hline MDR E. coli & $134(12.2)$ \\
\hline ESBL E. coli & $330(30)$ \\
\hline \multirow[t]{2}{*}{ Number of patients with $K$. pneumoniae enteric colonization } & 285 \\
\hline & ( $26 \%$ of total number of patients) \\
\hline MDR K. pneumoniae & $97(8.7)$ \\
\hline ESBL K. pneumoniae & $129(11.8)$ \\
\hline Number of patients colonized by MDR at presentation & $231(21)$ \\
\hline Number of patients colonized by ESBLO at presentation & 495 (41.9) \\
\hline Total number of febrile neutropenic episodes & 1461 \\
\hline Total blood culture positivity rate & $298 / 1461(20.4)$ \\
\hline Blood culture positivity rate in colonized patients & $253 / 736(34.4)$ \\
\hline
\end{tabular}

Abbreviations: ALL, acute lymphoblastic leukemia; AML, acute myeloid leukemia; CML, chronic myeloid leukemia; E. coli, Escherichia coli; ESBL, extended-spectrum $\beta$-lactamase; ESBLO, Extended-spectrum $\beta$-lactamase producing organism; HL, Hodgkin lymphoma; K. pneumoniae, Klebsiella pneumoniae; MDR, multidrug resistant; MDS, myelodysplastic syndromes; MRSA, methicillin-resistant S. aureus; NHL, non-HL; S. aureus, Staphylococcus aureus; VRE, vancomycin-resistant enterococci.

\section{Results}

In the 18-month study, 1094 patients with hematolymphoid malignancies were registered for treatment and had baseline rectal swab taken. The profile of bacterial isolates from rectal swab cultures is represented in - Table $\mathbf{1}$. The incidence of rectal swab colonization at baseline was $67.3 \%$ from 1094 patients. Escherichia coli was isolated in 680 patients, $19.7 \%$ of patients at baseline were colonized with MDR E. coli and 30\% were ESBLO. Klebsiella pneumoniae was isolated from 285 patients, $8.7 \%$ of patients were colonized with MDR K. pneumonia, while $11.8 \%$ were ESBLO. VRE was isolated in 32 patients. The profile of these patients is summarized in - Table 1.

During the study, 298 patients had positive blood cultures; of these, 253 had rectal colonization at baseline. These are summarized in - Table 2 .

The majority (57\%) of organisms causing BSI in our cohort were sensitive bacteria, while $43 \%$ were either MDR or ESBLO.
Table 2 Rectal colonization status and blood infection

\begin{tabular}{|l|l|l|}
\hline Isolate & Sensitivity & $\boldsymbol{n}(\%)$ \\
\hline BSI (positive cultures) $(n=298)$ & ESBLO & $\begin{array}{l}44 \\
(14.8)\end{array}$ \\
\hline & MDR & $\begin{array}{l}84 \\
(28.2)\end{array}$ \\
\hline & Sensitive & $\begin{array}{l}170 \\
(57)\end{array}$ \\
\hline $\begin{array}{l}\text { Rectal swab colonization in those } \\
\text { with positive blood culture }(n=253)\end{array}$ & ESBLO & 95 \\
\hline & MDR & $(31.9)$ \\
\hline & & 90 \\
& Sensitive & 68 \\
& & $(22.8)$ \\
\hline & No growth & $\begin{array}{l}45 \\
(15.1)\end{array}$ \\
\hline
\end{tabular}

Abbreviations: BSI, bloodstream infection; ESBLO, Extended-spectrum $\beta$-lactamase-producing organism; MDR, multidrug resistant 
Further analysis of the 253 patients with rectal colonization who developed BSI and their outcomes is illustrated in a Sankey diagram ( - Fig. 1).

At baseline, most of the patients (62.1\%) with confirmed BSI were colonized with either ESBLO (31.9\%) or MDR (30.2\%). Forty-five patients $(15.1 \%)$ were not colonized at baseline. We assessed the relationship between rectal colonization at baseline with confirmed BSI. For patients colonized with either MDR or ESBLO, the majority 116 (62.7\%) developed BSI caused by either MDR or ESBLO, while the remaining 37.3\% of them developed BSI caused by sensitive bacteria. In contrast, among patients without baseline colonization or colonized by sensitive bacteria, only 12 (10.6\%) of them developed BSI caused by either MDR or ESBLO, while most (89.4\%) developed BSI caused by sensitive organisms. These differences were statistically significant $(p<0.001)$. In other words, the baseline rectal swab had a sensitivity and a specificity of $90.6 \%$ and $59.4 \%$, respectively, in predicting BSI with either MDR or ESBLO (-Table 3).

Comparing MDR to ESBLO rectal colonization, the likelihood of patients colonized with ESBLO to develop sepsis due to ESBLO was $25.3 \%$, while that of those colonized with MDR to develop MDR sepsis was 51.1\% ( $p=0.049)$ (-Table 3).

Patients with either MDR or ESBLO colonization were more likely to be transferred to the ICU (49.7\%) as compared with those without baseline colonization or colonized by sensitive bacteria (1.8\%), as shown in - Table 4.

Patients colonized with MDR or ESBLO had an equal chance of ICU transfer ( - Table 4), but mortality was higher in the MDR group as compared with the ESBLO group (-Table 5).

Death was also more likely in those colonized with MDR or ESBLO at baseline compared with those who were not (-Table 5).

Table 3 Correlation of rectal colonization at baseline by MDR organisms and ESBLO with BSI caused by MDR organisms and ESBLO

\begin{tabular}{|c|c|c|c|c|c|}
\hline Rectal colonization at baseline $(n=298)$ & $n(\%)$ & \multicolumn{2}{|c|}{ BSI with MDR or ESBLO, $n(\%)$} & \multirow[t]{2}{*}{$p\left(\chi^{2}\right)$} & \\
\hline & & Yes & No & & \\
\hline Either & $185(62.1)$ & $116(62.7)$ & $69(37.3)$ & $<0.001(77.661)$ & \\
\hline Neither & $113(37.9)$ & $12(10.6)$ & $101(89.4)$ & & \\
\hline \multirow[t]{2}{*}{ Rectal colonization at baseline $(n=185)$} & $n$ & \multicolumn{3}{|c|}{ BSI } & $p\left(\chi^{2}\right)$ \\
\hline & & ESBLO & MDR & Sensitive & \\
\hline ESBLO & 95 & $24(25.3)$ & $34(35.8)$ & 37 (38.9) & $0.049(6.032)$ \\
\hline MDR & 90 & $12(13.3)$ & $46(51.1)$ & $32(35.6)$ & \\
\hline
\end{tabular}

Abbreviations: BSI, blood stream infection ESBLO, Extended-spectrum $\beta$-lactamase-producing organism; MDR, multidrug-resistant.

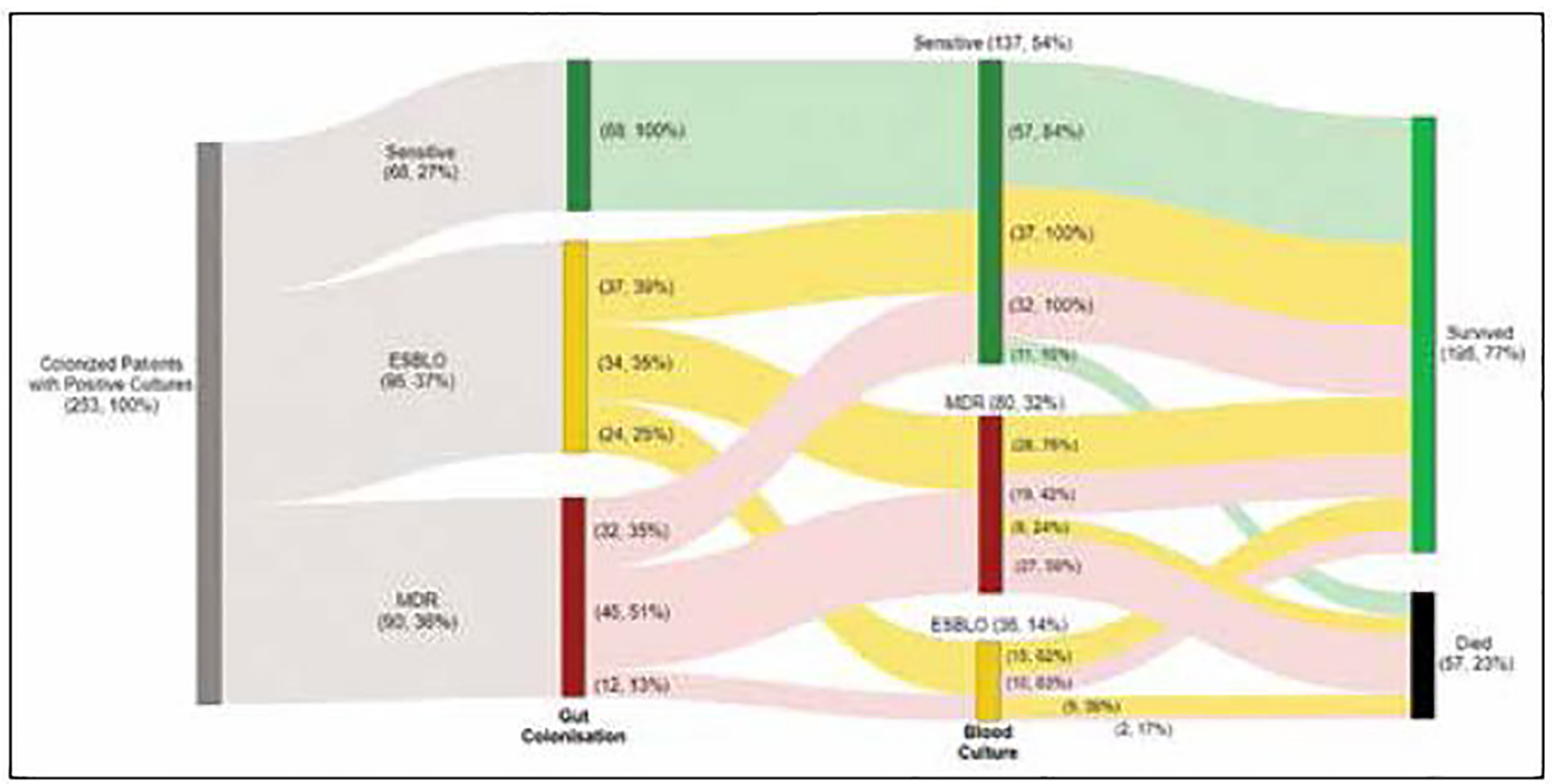

Fig. 1 Illustration of baseline rectal colonization and the development of blood stream infection (BSI) and outcome of infection. ESBLO: Extended-spectrum $\beta$-lactamase-producing organisms, MDR: multidrug resistant organisms. 
Table 4 Rectal colonization with MDR and ESBLO and likelihood of ICU admission

\begin{tabular}{|l|l|l|l|l|}
\hline $\begin{array}{l}\text { Rectal colonization at baseline }(\boldsymbol{n}=\mathbf{2 9 8}) \\
\text { MDR and ESBL }\end{array}$ & $\boldsymbol{n}(\%)$ & ICU admission, $\boldsymbol{n}(\%)$ & No ICU admissions, $\boldsymbol{n}(\%)$ & $\boldsymbol{p}\left(\boldsymbol{\chi}^{2}\right)$ \\
\hline Either & $185(62.1)$ & $92(49.7)$ & $93(50.3)$ & $111(98.2)(74.725)$ \\
\hline Neither & $113(37.3)$ & $2(1.8)$ & $51(53.7)$ & 0.001 \\
\hline ESBL & $95(51.4)$ & $44(46.3)$ & $42(46.7)(0.910)$ & 0.34 \\
\hline MDR & $90(48.6)$ & $48(53.3)$ & \\
\hline
\end{tabular}

Abbreviations: ESBL, extended-spectrum $\beta$-lactamase; ESBLO, extended-spectrum $\beta$-lactamase-producing organism; ICU, intensive care unit; MDR, multidrug-resistant.

Table 5 Rectal colonization with multidrug resistant and extended spectrum $\beta$-lactamase producing organism and likelihood of mortality

\begin{tabular}{|l|l|l|l|l|}
\hline $\begin{array}{l}\text { Rectal colonization at baseline }(\boldsymbol{n}=\mathbf{2 9 8}) \text { MDR and } \\
\text { ESBLO }\end{array}$ & $\boldsymbol{n}(\%)$ & Dead, $\boldsymbol{n}(\%)$ & Alive, $\boldsymbol{n}(\%)$ & $\boldsymbol{p}\left(\chi^{2}\right)$ \\
\hline Either & $185(62.1)$ & $46(24.9)$ & $139(75.1)$ & $0.001(10.382)$ \\
\hline Neither & $113(37.9)$ & $11(9.7)$ & $102(90.3)$ & \\
\hline ESBLO & $95(51.4)$ & $17(17.9)$ & $78(82.1)$ & $0.024(5.078)$ \\
\hline MDR & $90(48.6)$ & $29(32.2)$ & $61(67.8)$ & \\
\hline
\end{tabular}

Abbreviations: ESBLO, extended spectrum $\beta$-lactamase producing organisms; MDR, multidrug resistant.

\section{Discussion}

Recent reports from our institution showed the high incidence of both MDR and ESBLO in BSI compared with what is being reported in Western literature.,6,7 Another study from our institute also reported an alarming incidence of MDR bacteria in rectal surveillance cultures of pediatric patients, $58.4 \%$ and $20.2 \%$ for ESBL and CRE, respectively, thus further strengthening the call for institutions to review antibiotic usage policy. ${ }^{8}$

Antibiotic stewardship, institutional antibiotic usage, and infection prevention and control have gained prominence in recent times as the world is faced with an increasing spate of drug-resistant organisms without any appreciable increase in the development of new antimicrobials to combat this pandemic. ${ }^{2}$ The focus has now shifted to the judicious use of antimicrobials with emphasis on early commencement of appropriate empirical antimicrobials while awaiting culture reports in immunosuppressed patients. It has been demonstrated in reports that organisms invading the bloodstream following commencement of cancer treatment with its attendant effect on breaching the integrity of the gastrointestinal mucosal barrier has led to translocation of pathogens colonizing the gut into the bloodstream consequently, leading to sepsis. ${ }^{2,9-11}$ Enteric colonization by drug-resistant pathogens has therefore been named in ECIL-4 guidelines as the most important risk factor for infection by MDR bacteria, with special emphasis on ESBLO, CRE, resistant nonfermenters, methicillin-resistant Staphylococcus aureus (MRSA) with mic $\geq 2 \mathrm{mg} / \mathrm{L}$ and VRE.

Several other reports and guidelines such as the Indian Council of Medical Research (ICMR) have informed the need to have surveillance cultures to guide the choice of empirical antibiotics for the management of patients with febrile neutropenia. ${ }^{2,12-16}$ However, some do not recommend this strategy, owing to contradictory or inconclusive evidence to support enteric colonization as a source of clinical infections in the immunocompromised host. In this context, a retrospective review of 794 allogeneic hematopoietic transplant patients had found only 12 patients developing MDR Pseudomonas infections, out of which only about half had prior colonization, indicating that there was another source for the infection. Fluoroquinolone prophylaxis was however administered to $83 \%$ of this cohort, which may have influenced the outcome. ${ }^{17}$ Although a few studies are inconclusive on the utility of surveillance cultures, these have major limitations such as inadequate sample size and prior utilization of antimicrobial prophylaxis. There is however a consistent pattern of increased morbidity, higher overall mortality, and increased cost of care in patients who develop infections caused by drug-resistant pathogens without timely choice of appropriate empirical antimicrobials. . $, 10,18$

The correlation between gut colonization of resistant pathogens and their subsequent implication in BSI in a pediatric hematolymphoid cohort is being reported in a low-middle income country, with a high prevalence of drug-resistant pathogens ( - Tables 2, 3 and - Fig. 1), that has utilized the ECIL-4 guidelines.

In an observational prospective cohort of 126 adult patients with hematolymphoid malignancies in Mexico, it was found that colonization with ESBL-E. coli increased the risk of BSI by the same strain, had a shorter time to death, longer hospital stay, and higher infection-related costs. ${ }^{10}$ This was corroborated in our study as patients with either MDR or ESBLO colonization were more likely to be transferred to the ICU (49.7\%) compared with those without baseline colonization or colonized by sensitive bacteria (1.8\%), and this was associated with a higher mortality, especially in the patient with MDR colonization ( - Fig. 1 and - Tables 4 and 5 ). This 
was found in an earlier study among pediatric patients in our institution and strengthened the call for early suspicion of sepsis and appropriate upfront use of high-grade antibiotics in this group of immunosuppressed patients with MDR colonization to curtail these adverse outcomes.

VRE colonization did not lead to an increased incidence of BSI by the same suggestion that our center can continue to withhold initial coverage for VRE in those colonized with this pathogen, as the literature is still inconclusive with regard to initial coverage for VRE due to its low pathogenicity. ${ }^{19,20}$

\section{Conclusions}

We conclude that a high prevalence of MDR colonization at presentation significantly results in MDR BSI, which further results in a significant increase in ICU admissions and mortality. This would justify the use of a "de-escalation" antibiotic policy as defined by ECIL4 recommendations. Whether such a strategy has been successful in impacting outcomes, would need further study.

\section{Financial Support and Sponsorship}

Nil.

\section{Conflicts of Interest}

There are no conflicts of interest.

\section{Acknowledgments}

We gratefully acknowledge the contributions of former Group Member, Dr. Brijesh Arora, for the groundwork and vision for many of the supportive care policies analyzed directly or indirectly in this study. We are grateful to Dr. Kokou Amegan-Aho and Mr. Emmanuel Asimadi for their respective roles in proofreading the revised manuscript.

\section{References}

1 Radhakrishnan V, Vijaykumar V, Ganesan P, Rajendranath R, Trivadi G, Tenali S. Bloodstream infections in pediatric patients at Cancer Institute, Chennai. Indian J Cancer 2014;51(4):418-419

2 Averbuch D, Orasch C, Cordonnier C, et al. ECIL4, a joint venture of EBMT, EORTC, ICHS, ESGICH/ESCMID and ELN. European guidelines for empirical antibacterial therapy for febrile neutropenic patients in the era of growing resistance: summary of the 2011 4th European Conference on Infections in Leukemia. Haematologica 2013;98(12):1826-1835

3 Magiorakos AP, Srinivasan A, Carey RB, et al. Multidrugresistant, extensively drug-resistant and pandrug-resistant bacteria: an international expert proposal for interim standard definitions for acquired resistance. Clin Microbiol Infect 2012;18(3):268-281

4 Paterson DL, Bonomo RA. Extended-spectrum beta-lactamases: a clinical update. Clin Microbiol Rev 2005;18(4):657-686

5 Thacker N, Pereira N, Banavali SD, et al. Epidemiology of blood stream infections in pediatric patients at a tertiary care cancer centre. Indian J Cancer 2014;51(4):438-441

6 Prabhash K, Medhekar A, Ghadyalpatil N, et al. Blood stream infections in cancer patients: a single center experience of isolates and sensitivity pattern. Indian J Cancer 2010;47(2):184-188

7 Mikulska M, Viscoli C, Orasch C, et al. Fourth European Conference on Infections in Leukemia Group (ECIL-4), a joint venture of EBMT, EORTC, ICHS, ELN and ESGICH/ESCMID. Aetiology and resistance in bacteraemias among adult and paediatric haematology and cancer patients. J Infect 2014;68(4):321-331

8 Thacker N, Pereira N, Banavali SD, et al. Alarming prevalence of community-acquired multidrug-resistant organisms colonization in children with cancer and implications for therapy: A prospective study. Indian J Cancer 2014;51(4):442-446

9 Ford CD, Lopansri BK, Haydoura S, et al. Frequency, risk factors, and outcomes of vancomycin-resistant Enterococcus colonization and infection in patients with newly diagnosed acute leukemia: different patterns in patients with acute myelogenous and acute lymphoblastic leukemia. Infect Control Hosp Epidemiol 2015;36(1):47-53

10 Cornejo-Juárez P, Suárez-Cuenca JA, Volkow-Fernández P, et al. Fecal ESBL Escherichia coli carriage as a risk factor for bacteremia in patients with hematological malignancies. Support Care Cancer 2016;24(1):253-259

11 Van Leeuwen PA, Boermeester MA, Houdijk AP, et al. Clinical significance of translocation. Gut 1994;35(1(Suppl):S28-S34

12 Bassetti M, Righi E. Multidrug-resistant bacteria: what is the threat? Hematology (Am Soc Hematol Educ Program) 2013;2013:428-432

13 Kang CI, Chung DR, Ko KS, Peck KR, Song JH; Korean Network for Study of Infectious Diseases. Risk factors for infection and treatment outcome of extended-spectrum $\beta$-lactamase-producing Escherichia coli and Klebsiella pneumoniae bacteremia in patients with hematologic malignancy. Ann Hematol 2012;91(1):115-121

14 Gudiol C, Calatayud L, Garcia-Vidal C, et al. Bacteraemia due to extended-spectrum beta-lactamase-producing Escherichia coli (ESBL-EC) in cancer patients: clinical features, risk factors, molecular epidemiology and outcome. J Antimicrob Chemother 2010;65(2):333-341

15 Shankar K, Radhakrishnan V, Vijayakumar V, et al. Prevalence of multi-drug resistant organisms in stool of paediatric patients with acute leukaemia and correlation with blood culture positivity: A single institution experience. Pediatr Blood Cancer 2018;65(1):e26740

16 Indian Council for Medical Research. Department of Health Research Treatment Guidelines for Antimicrobial use in Common Syndromes. Available at: https://www.icmr.nic.in/ sites/default/files/guidelines/Treatment_guidelines_2017.pdf. Accessed July 10, 2017

17 Nesher L, Rolston KV, Shah DP, et al. Fecal colonization and infection with Pseudomonas aeruginosa in recipients of allogeneic hematopoietic stem cell transplantation. Transpl Infect Dis 2015;17(1):33-38

18 Gudiol C, Ayats J, Camoez M, et al. Increase in bloodstream infection due to vancomycin-susceptible Enterococcus faecium in cancer patients: risk factors, molecular epidemiology and outcomes. PLoS One 2013;8(9):e74734

19 Vergis EN, Hayden MK, Chow JW, et al. Determinants of vancomycin resistance and mortality rates in enterococcal bacteremia. a prospective multicenter study. Ann Intern Med 2001;135(7):484-492

20 DiazGranados CA, Jernigan JA. Impact of vancomycin resistance on mortality among patients with neutropenia and enterococcal bloodstream infection. J Infect Dis 2005;191(4):588-595 